\title{
Computer generation of bivariate normal-curve values
}

\author{
LARRY D. EVANS \\ University of Arkansas for Medical Sciences, Little Rock, Arkansas
}

\begin{abstract}
As psychological assessment broadens and becomes increasingly based on multiple sources of data, univariate normal-curve tables become less and less useful in determining the prevalence and severity of clinical disorders. Multivariate normal-curve data are required. The Visual Basic based Bivariate Agreement Program allows users to obtain percentages of the bivariate normal distribution at various correlations and univariate $z$-score values. This computer program simulates a bivariate distribution of up to 75,000 cases, and reports the percentage of cases above, below, and outside the $z$-scores. Program function and simulation integrity are discussed, and an application illustrates program use.
\end{abstract}

Univariate normal-curve tables have been useful to clinical psychology in defining target rates, determining severity, or assessing the incidence of a single attribute. However, as clinical assessment broadens and decision making becomes increasingly based on multiple measures, these tables become increasingly less useful. Multivariate normal-curve tables are needed to integrate and interpret the results from two or more measures of normally distributed attributes.

Although univariate normal-curve values are generally presented in a printed table, such tables are impractical for use with multivariate normal-curve values. A separate table would be required not only for every possible correlation between the variables, but also for every combination of intersecting vectors within the distribution. As a result, the only published multivariate data are bivariate expectancy tables (e.g., Schrader, 1965; Schyerger \& Emerson, 1981) which provide general expectancies for only individual scores and, in particular, for the likelihood of obtaining a specific criterion score given a predictor score and the correlation between scores.

This paper extends the expectancy tables to the entire bivariate normal distribution by describing a computer program, the Bivariate Agreement Program, which simulates the distribution. Users enter the correlation coefficient between variables of two univariate distributions, the $z$-score vectors of interest that intersect within the bivariate distribution, and the number of data points to include in the simulation (up to 75,000). Results show the percentage of data points in each of the four quadrants formed by the vectors. Two of the quadrants represent percentage agreement that data points are below or above both $z$ scores. The other quadrants represent percentage disagreement when only one variable's value falls below or above its $z$ score. Clinically, agreement is of primary

Correspondence should be addressed to L. D. Evans, Dennis Developmental Center, 1612 Maryland Street, Little Rock, AR 72202 (email: levans@ddcchild.ach.uams.edu). importance, as it provides the information needed to determine the percentage of a normal population that, when given two measures, would fail or pass both measures at given $z$-score cutoffs.

The importance of agreement with a bivariate distribution is further illustrated in such cases as the determination of the incidence of a disorder identified by a pair of diagnostic measures (e.g., adaptive behavior and intelligence for identification of mental retardation), the prediction of the number of scores failing or passing one or both measures used to identify a population (e.g., percentage of applicants surviving a pair of screening measures), and the selection of cutoff values to identify a predetermined percentage of the population on the basis of scores from a pair of measures (e.g., score cutoffs needed to target an intervention or research population).

Although it is possible to calculate bivariate normalcurve density values with integral calculus, it is quite difficult to find correspondence of the values from the threedimensional bivariate normal distribution with the original univariate distributions. For example, a clinician may administer intelligence and adaptive behavior tests to determine if concurrent deficits in functioning exist. While a bivariate score of 75,65 may be severe and fall in the outer $3 \%$ of scores in the bivariate distribution, the score of 75 does not fall in the lowest $3 \%$ of scores in its univariate distribution. As a result, computer simulation is needed to generate bivariate distributions so that agreement rates at varying univariate cutoff indices can be determined. Fortunately, computers have demonstrated the ability to simulate sufficient numbers of scores to closely approximate true values (Burns, 1984; Macmann \& Barnett, 1985), and even current microcomputers provide ample speed and memory for adequate simulation.

\section{BIVARIATE AGREEMENT PROGRAM}

\section{Hardware Requirements}

The Bivariate Agreement Program is written in Version 1.0 of Visual Basic for DOS. A 486 or faster proces- 
sor is recommended in order to generate sufficient numbers of data points for accurate and timely simulation. Run time for a single simulation of all 75,000 data points is slightly more than $5 \mathrm{~min}$ for a Pentium $90-\mathrm{MHz}$ processor. The compiled program and data files are contained on, and can be executed from, a single, high-density floppy diskette.

\section{Program Function}

The program's input options and results are presented on a single form. The user enters the correlation between variables, a $z$-score cutoff index for each variable, and the number of data points (up to 75,000) to include in the bivariate normal distribution simulation. The program then performs these steps:

1. A univariate normal-curve value from a sequential file of randomized normal-curve values is entered. This file was generated as a nonrandom file of 75,000 normalcurve values, then reentered into the file in a random order. It is necessary to create an initial nonrandom file in order to ensure that the distribution is as close to normal as possible, a task made difficult by the curvilinear distribution and its irrational components (e and pi). The integrity of the generated normal-curve values was checked by comparing them with the expected values from published univariate tables. Table 1 shows the results of the integrity check; the generated percentages match the expected normal-curve percentages to two decimal places. The randomized normal-curve values simulate the normal curve more accurately than did previous efforts to directly generate random normal-curve values (Evans, 1992). The randomized normal-curve value entered in this step becomes the data point for Variable 1 .

2. The standard deviation of differences between the scores of Variable 1 and Variable 2 is calculated on the basis of the correlation between scores. A second randomized normal-curve value is multiplied by the standard deviation of differences to obtain the data point for Variable 2. This step allows for the selection of a second random value while preserving the correlation between variables.

3 . The two data points form a single bivariate score which is compared with the $z$ scores for each variable. Figure 1 provides an example of the shape of the bivariate

Table 1

Comparison of Computer-Generated and Actual Normal-Curve Values

\begin{tabular}{|c|c|c|}
\hline \multicolumn{3}{|c|}{ Normal-Curve Values } \\
\hline \multirow[b]{2}{*}{ Normal-Curve Area $(S D)$} & \multicolumn{2}{|c|}{ Normal-Curve Percentages } \\
\hline & $\begin{array}{l}\text { Expected } \\
\text { Values* }\end{array}$ & $\begin{array}{c}\text { Generated } \\
\text { Values } \dagger \\
\end{array}$ \\
\hline $3.0 \leq x$ & .13499 & .13466 \\
\hline $2.5 \leq x<3.0$ & .48598 & .48599 \\
\hline $2.0 \leq x<2.5$ & 1.65404 & 1.65398 \\
\hline $1.5<x<2.0$ & 4.40571 & 4.40528 \\
\hline $1.0<x<1.5$ & 9.18481 & 9.18521 \\
\hline $0.5<x<1.0$ & 14.98822 & 14.98780 \\
\hline $0.0<x<0.5$ & 19.14625 & 19.14641 \\
\hline
\end{tabular}

*Based on univariate tables (Edwards, 1954). †Chi square goodness of fit: $\chi^{2}(6, N=75,000)=.0007, p<.0001$.

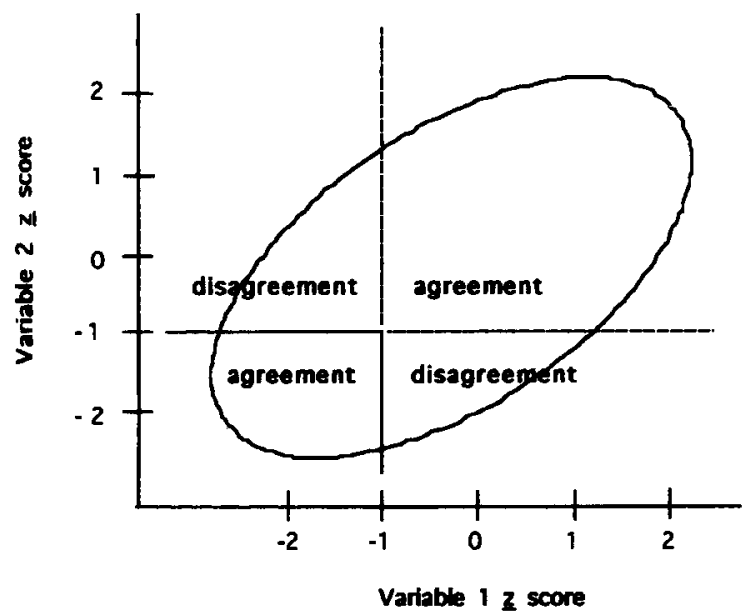

Figure 1. Bivariate distribution and the quadrants showing agreement and disagreement that cases fall above or below the shared $z$ score of $-\mathbf{1 . 0 0}$.

distribution and the areas formed by the entered $z$-score vectors. As shown, percentages of agreement and disagreement result with respect to the $z$ scores.

4. Because the bivariate distribution is symmetric, the program reverses the signs of the $z$ scores and compares the bivariate score with a second pair of $z$ scores. For example, the percentage of bivariate scores that falls below a $z$-score cutoff of -1 for each variable should equal the percentage that falls above a cutoff of +1 for each variable.

5. The program repeats the above steps for the entered number of data points and counts the number of data points falling in each quadrant shown in Figure 1. The results of Step 4 are averaged with those of Step 3, effectually doubling the number of simulated data points.

\section{Program Output}

The results of the Bivariate Agreement Program are presented on the computer screen. First, the percentage of bivariate data points below both $z$ scores is shown; then the percentage above both $z$ scores is shown. Next, the percentage of instances in which Variable 1 was below and Variable 2 was above their respective $z$ scores is shown, followed by the percentage of instances in which Variable 1 was above and Variable 2 was below their respective $z$ scores. Finally, the program reports the correlation coefficient for the simulation. Table 2 shows the results for simulations at the correlation coefficient of .50. The table shows results for only a few $z$-score intervals at a single correlation, further illustrating the inherent impracticality of printed bivariate tables.

\section{Application}

To apply the Table 2 values, consider an evaluator or researcher with a single measure intending to screen an attribute that is normally distributed in a population. With a cutoff score of $\leq-1.50$ standard deviations for failure, a yield of $6.68 \%$ is expected on the basis of uni- 
Table 2

Bivariate Normal Distribution Percentages at .50 Correlation

\begin{tabular}{|c|c|c|c|c|c|c|c|}
\hline \multirow{2}{*}{$\begin{array}{c}\text { Variable } 1 \\
(z \text { score })\end{array}$} & \multicolumn{7}{|c|}{ Variable 2 ( $z$ single score $)$} \\
\hline & .00 & -.50 & -1.00 & -1.50 & -2.00 & -2.50 & -3.00 \\
\hline .00 & $\begin{array}{l}33.34 \\
33.34 \\
16.66 \\
16.66\end{array}$ & $\begin{array}{r}22.76 \\
41.78 \\
27.24 \\
8.22\end{array}$ & $\begin{array}{r}12.77 \\
46.90 \\
37.23 \\
3.10\end{array}$ & $\begin{array}{r}5.70 \\
49.08 \\
44.30 \\
.92\end{array}$ & $\begin{array}{r}2.06 \\
49.79 \\
47.94 \\
.21\end{array}$ & $\begin{array}{r}.58 \\
49.96 \\
49.42 \\
.00\end{array}$ & $\begin{array}{r}.13 \\
50.00 \\
49.87 \\
.00\end{array}$ \\
\hline-.50 & & $\begin{array}{l}16.43 \\
54.49 \\
14.54 \\
14.54\end{array}$ & $\begin{array}{r}9.79 \\
62.95 \\
21.18 \\
6.08\end{array}$ & $\begin{array}{r}4.64 \\
67.05 \\
26.33 \\
1.98\end{array}$ & $\begin{array}{r}1.76 \\
68.53 \\
29.21 \\
.50\end{array}$ & $\begin{array}{r}.52 \\
68.93 \\
30.45 \\
.01\end{array}$ & $\begin{array}{r}.12 \\
69.01 \\
30.85 \\
.01\end{array}$ \\
\hline-1.00 & & & $\begin{array}{r}6.28 \\
74.54 \\
9.59 \\
9.59\end{array}$ & $\begin{array}{r}3.19 \\
80.71 \\
12.67 \\
3.42\end{array}$ & $\begin{array}{r}1.32 \\
83.19 \\
14.55 \\
.94\end{array}$ & $\begin{array}{r}.42 \\
83.93 \\
15.45 \\
.20\end{array}$ & $\begin{array}{r}.10 \\
84.10 \\
15.77 \\
.03\end{array}$ \\
\hline-1.50 & & & & $\begin{array}{r}1.78 \\
88.54 \\
4.84 \\
4.84\end{array}$ & $\begin{array}{r}.81 \\
91.92 \\
5.81 \\
1.46\end{array}$ & $\begin{array}{r}.30 \\
93.04 \\
6.34 \\
.34\end{array}$ & $\begin{array}{r}.08 \\
93.32 \\
6.54 \\
.06\end{array}$ \\
\hline-2.00 & & & & & $\begin{array}{r}.41 \\
95.88 \\
1.86 \\
1.86\end{array}$ & $\begin{array}{r}.16 \\
97.28 \\
2.11 \\
.46\end{array}$ & $\begin{array}{r}.05 \\
97.65 \\
2.21 \\
.08\end{array}$ \\
\hline-2.50 & & & & & & $\begin{array}{r}.07 \\
98.83 \\
.55 \\
.55\end{array}$ & $\begin{array}{r}.02 \\
99.27 \\
.59 \\
.11\end{array}$ \\
\hline-3.00 & & & & & & & $\begin{array}{r}.01 \\
99.74 \\
.12 \\
.12\end{array}$ \\
\hline
\end{tabular}

Note-The top number in each group denotes the percentage of bivariate cases below each $z$-score cutoff. The second number indicates the percentage above both cutoffs. The third and fourth values show the percentage below the Variable 1 cutoff and above the Variable 2 cutoff and above the Variable 1 cutoff and below the Variable 2 cutoff, respectively. These percentages are based on a Bivariate Agreement Program simulation with 75,000 cases.

variate tables. The yield is unknown, however, when a second, complementary measure (correlation $=.50$ with first measure) is added to improve the screening. If failure is defined as scores of $\leq-1.50$ on both measures, then, according to Table 2, a yield of approximately $1.78 \%$ will be obtained, as only about $1.78 \%$ of normally distributed scores are at or below a univariate $z$-score cutoff of -1.50 on both measures. The evaluator can increase the yield by raising the cutoff to -1.00 for each measure and, according to Table 2, approximately $6.28 \%$ can be expected to fail both measures. If the cutoff value of -1.50 was retained for both measures but failure was defined as one or both scores $\leq-1.50$, then Table 2 indicates agreement for $1.78 \%$ and disagreement for $4.84 \%$ $+4.84 \%$ of cases, resulting in a total yield of approximately $11.46 \%$.

Although results from the Bivariate Agreement Program may be helpful for group data, normal-curve percentages, univariate or multivariate, must be interpreted for individual cases with respect to measurement error (Braden \& Algina, 1989). Such consideration for measurement error can provide information regarding probability in individual cases. Significant measurement error can be magnified when bivariate values are considered, yielding "hit" rates near chance levels (Accardo \& Whitman, 1991). Instruments with higher reliability, clinical judgment, and additional sources of information should be considered.

\section{Availability}

A copy of the compiled program and data files is available from the author upon receipt of a self-addressed, postage-paid mailer and one DOS-formatted, high-density floppy disk.

\section{REFERENCES}

ACCARDO, P. J., \& WhITMAN, B. Y. (1991). The misdiagnosis of the hyperactive child. In P. J. Accardo, T. A. Blondis, \& B. Y. Whitman, Attention deficit disorders and hyperactivity in children. New York: Marcel Dekker.

Braden, J. P., \& Algina, J. (1989). A method for determining the probability of special education eligibility. Journal of School Psychology, 27, 5-13.

BURNs, E. (1984). The bivariate normal distribution and the IQ of learning disability samples. Journal of Learning Disabilities, 17, 294-295.

EDWARDS, A. L. (1954). Statistical methods for the behavioral sciences. New York: Holt.

Evans, L. D. (1992). Standard score analysis (Version 1.1) [Computer software]. North Little Rock, AR: WtL.

MACMANN, G. M., \& BARNETT, D. W. (1985). Discrepancy score analysis: A computer simulation of classification stability. Journal of Psychoeducational Assessment, 3, 363-375.

SCHRADER, W. B. (1965). A taxonomy of expectancy tables. Journal of Educational Measurement, 2, 29-35.

SChyerger, J. M., \& EMERSON, P. (1981). Bridges the gap between correlation coefficient and client general expectancy table. Multivariate Experimental Clinical Research, 5, 73-77.

(Manuscript received November 20, 1995; accepted for publication January 3, 1996.) 